\title{
Factores que predisponen a la elevación de la tensión arterial en pacientes normotensos operados en cirugía electiva
}

\author{
Factors predisposing to elevated blood pressure in normotensive patients operated on in \\ elective surgery
}

Dania Yusimí Pompa Rodríguez ${ }^{1}$, Víctor José Vasallo Comendeiro ${ }^{2}$ y Alberto Pompa Núñez ${ }^{3}$

DOI. 10.21931/RB/2020.05.02.6

Resumen: La estabilidad circulatoria es uno de los propósitos principales durante el acto anestésico-quirúrgico. Por eso, el objetivo propuesto fue determinar la relación de los factores de riesgo identificados, con la elevación de la tensión arterial en pacientes normotensos operados en cirugía electiva, bajo anestesia general orotraqueal. Se realizó un estudio de caso-control con $n=88$ normotensos que fueron operados en el transcurso del año 2018, distribuidos normalmente $(p=0,000)$ en compensados $(n=44)$ y descompensados ( $n=44)$. Se analizaron variables: anestésico-quirúrgicas, factores de riesgo (independientes) y variable dependiente: descompensación hemodinámica (dicotómica). Se realizaron pruebas de hipótesis: Kolmogorov-Smirnov (normalidad), ONEWAY (diferencias tensionales entre grupos) y de asociación: Ji-cuadrado, Fisher y Regresión logística ( $p<0,05)$ y Odds Ratio $(O R>1)$. Los resultados fueron representados en tablas y gráficos estadísticos y a través del método de análisis y síntesis fueron expresados textualmente. Se concluye que los antecedentes patológicos personales y familiares, el tabaquismo, cafeísmo, sedentarismo y el hipercolesterolemia constituyen factores de riesgo de hipertensión arterial, que en la medida que se conjugan se produce una potenciación de sus efectos y se elevan considerablemente los riesgos de los pacientes operados en cirugía electiva, aun cuando estos sean considerados como normotensos en la evaluación preoperatoria y al entrar al salón.

Palabras clave: Hipertensión arterial, factores de riesgo, cirugía electiva, anestesia.

Abstract: Circulatory stability is one of the main factors during the anesthetic-surgical act. Therefore, the proposed objective was to determine the relationship of the identified risk factors with the derivation of blood pressure in normotensive patients operated on in elective surgery under general orotracheal anesthesia. A case-control study was performed with $n=88$ normotensive patients who underwent surgery in 2018, normally distributed $(p=0.000)$ is compensated $(n=44)$ and decompensated $(n=44)$. Variables were analyzed: anesthetic-surgical, risk factors (independent), and dependent variable: hemodynamic decompensation (dichotomous). Hypothesis tests were performed: Kolmogorov - Smirnov (normality), ONEWAY (blood pressure differences between groups), and association: Chi-square, Fisher, and Logistic regression $(p<0.05)$ and Odds Ratio $(O R>1)$. The results were represented in statistical tables and graphs, and through the analysis and synthesis method, they were expressed verbatim. It is concluded that personal and family pathological history, smoking, caffeine, sedentary lifestyle and hypercholesterolemia are risk factors for high blood pressure. These combinations produce a potency of their effects and increase the risks of the patients, operated in elective surgery, even when they are treated as normotensive in the preoperative evaluation and upon entering the ward.

Key words: Arterial hypertension, factors of risk, elective surgery, anesthetizes.

\section{Introducción}

La hipertensión arterial (HTA) es una enfermedad de etiología múltiple caracterizada por elevación persistente de la presión arterial sistólica, diastólica o de ambas, representa por sí misma una enfermedad, como también un factor de riesgo importante para otras enfermedades, fundamentalmente para la cardiopatía isquémica, insuficiencia cardiaca, enfermedad cerebro-vascular, insuficiencia renal y para la retinopatía ${ }^{1,2}$. Para el año 2014 se estimó que más del 20\% de los adultos mayores de 18 años, en el mundo, eran hipertensos y casi 10 millones de muertes fueron causadas por $\mathrm{HTA}^{3}$. A nivel mundial, afecta a más de mil millones de personas y aunque puede diagnosticarse y tratarse de forma satisfactoria, sólo una de cada siete personas hipertensas tiene la presión arterial controlada ${ }^{4}$.

La HTA es el factor de riesgo que está presente en la mayoría de las muertes causadas por enfermedades circulatorias, enfermedades coronarias y vascular encefálicas. En la mayor parte de las regiones existe una prevalencia del 15 al $30 \%$ de la enfermedad. En Cuba, en el año 2015, la tasa de prevalencia de HTA fue de 217,5 por 1000 habitantes, siendo el sexo femenino el más afectado con una tasa de 236,5 por 1000 habitantes, así como el grupo etario de 60 a 64 años $^{5}$.

La administración de anestesia puede suponer un riesgo para el paciente superior al de la cirugía y por eso la valoración preanestésica es un paso crucial que puede afectar a la seguridad clínica e integridad de todo el proceso quirúrgico. La aparición de HTA está generalmente condicionada a la existencia de uno o más factores predisponentes que son los mismos que contribuyen a una mayor morbilidad y mortalidad cardiovascular. Se resumen en: tabaquismo, sobrepeso, vida sedentaria, falta de ejercicios, ingesta excesiva de alcohol e hipercolesterolemia. Se añade la exagerada ingesta de sal ${ }^{6-10}$.

La estabilidad circulatoria es uno de los elementos principales en el perioperatorio de pacientes de alto riesgo. Por eso, 
el objetivo propuesto en esta investigación fue determinar la relación de los factores de riesgo identificados, con la elevación de la tensión arterial en pacientes normotensos operados en cirugía electiva, bajo anestesia general orotraqueal.

\section{Materiales y métodos}

\section{Diseño de la investigación}

Se realizó un estudio descriptivo observacional de caso-control, donde el universo estuvo conformado por 239 pacientes con sus correspondientes historias clínicas. Los mismos se caracterizaban por no ser hipertensos y fueron intervenidos con cirugía abdominal electiva, bajo anestesia general orotraqueal, a lo largo de un año en el Instituto Superior de Medicina Militar "Dr. Luis Díaz Soto" de La Habana.

A través del programa Epidat versión 3.1 se efectuó el diseño muestral y se obtuvo $n=88$ que se distribuyó equitativamente a razón de 1:1, correspondiente a: Grupo 1 pacientes normotensos descompensados, $\mathrm{n}=44$ y grupo 2 pacientes normotensos compensados, $n=44$, por intervención del muestreo simple aleatorio.

\section{Criterios de inclusión}

Pacientes no hipertensos que expusieron su consentimiento para la obtención de las variables clínicas y de laboratorio especificadas en el trabajo.

\section{Criterios de Exclusión}

Pacientes con hipertensión arterial conocida o de debut y los que no manifestaron interés en formar parte del estudio.

Se clasificaron como normotensos a los pacientes con cifras tensionales iguales o menores a $140 / 90 \mathrm{mmHg}^{3,7,10,11}$ y mayores a 90/60 mmHg e hipotensión cuando era inferior a 90/60 mmHg ${ }^{12,13}$. Estos pacientes no tenían antecedentes conocidos de la enfermedad y durante las consultas realizadas no se detectaron cifras de HTA. La cifra de TA se monitoreó en la consulta interna, al arribo al salón, a la entrada al quirófano y durante el acto anestésico-quirúrgico cada intervalo de 10 minutos.

\section{Variables anestésico-quirúrgicas}

Se contemplaron la tensión arterial y los factores de riesgo que condicionan su elevación, clasificados en modificables: Indice de Masa Corporal (IMC) $)^{14,15}$, hábito de fumar, el consumo de café, de bebidas alcohólicas, de sal, la hipercolesterolemia, el sedentarismo, el estrés y la ansiedad. No modificables: Edad, apariencia racial, sexo, antecedentes patológicos personales (APP) relacionados con eventos mórbidos crónicos anteriores al ingreso, antecedentes familiares (APF) de HTA y si estuvo compensado o descompensado durante el tiempo quirúrgico.

\section{Procedimiento anestésico empleado en el estudio}

Con todos los pacientes se siguió la misma metodología según lo normado en el Centro hospitalario para los que son intervenidos quirúrgicamente con anestesia general endotraqueal: Consulta interna el día antes de la intervención quirúrgica y el día de la operación, fueron trasladados en camillas al departamento de preanestesia. La premedicación fue con benzodiacepinas (midazolán $0.15 \mathrm{mg} / \mathrm{kg}$ ), se realizó un registro de tensión arterial y de frecuencia cardiaca, antes y después de la premedicación. En el salón se les monitoreó el electrocardiograma, la tensión arterial (TA) sistólica, diastólica y media, la frecuencia cardiaca (FC) y la oximetría de pulso $\left(\mathrm{SO}_{2}\right)$. La in- ducción anestésica se inició con la administración endovenosa (EV) de Tiopental con una dosis de $5 \mathrm{mg} / \mathrm{kg}$, fentanil $5 \mathrm{mcg} / \mathrm{kg}$, Vecuronio 0,1 mg/kg y lidocaína $2 \%$ a $1 \mathrm{mg} / \mathrm{kg}$. Se conectó el flujo de gases anestésicos, óxido nitroso, con una $\mathrm{FiO}_{2}$ de 0,4 y el halotano en correspondencia con las necesidades individuales de cada paciente. En el transoperatorio se repusieron los egresos con cristaloides y coloides según lo calculado individualmente.

\section{Análisis Estadístico}

Una vez finalizado el acto quirúrgico se recopilaron los datos correspondientes al acto anestésico quirúrgico en las HC para su procesamiento estadístico. Con la información recolectada se creó una base de datos en el programa SPSS 11.5.1 y se analizó mediante técnicas de estadística, descriptiva e inferencial paramétrica. Previamente se determinó el tipo de distribución que siguió la muestra por medio de la prueba Kolmogorov -Smirnov que resultó normal, $\mathrm{p}<\mathrm{a}$.

\section{Resultados y discusión}

En la Tabla 1, se observa que en el período preanestésico los normotensos descompensados hemodinámicamente durante el transquirúrgico (TQ) presentaron mayores valores de presión sistólica $(p=0,034)$ y diastólica $(p=0,001)$ que los normotensos que se mantuvieron compensados. Se observa que, aun siendo los valores de TA correspondiente a cada grupo normales, este hecho no constituye una garantía absoluta para que el transoperatorio transcurra sin riesgos.

Otros investigadores han referido que el acto anestésico quirúrgico representa un factor considerablemente estresante, por lo que muchas veces resulta impredecible la posible respuesta del paciente ${ }^{16,17,18}$. Se han investigado pacientes con antecedentes de haber elevado sus cifras de presión arterial ante situaciones como acudir al consultorio médico y la obtención de cifras tensionales normales en casa, es lo que se denomina "hipertensión de bata blanca"10. Una prueba de estimulación por frío en 30 pacientes dio como resultado que 24 experimentaron hipertensión arterial. A estos pacientes les fue indicado tratamiento previo y a los 6 negativos no se les prescribió tratamiento por no expresar hiperreactividad. Se obtuvo como resultado que no se produjera hipertensión perioperatoria, ni suspensiones del acto quirúrgico; concluyéndose que esta prueba es un valioso instrumento para la predicción tensional transoperatoria y auxiliar en la decisión de prescribir hipotensores, como medicación previa ${ }^{19,20}$.

En la Tabla 2, se observa que, aunque en el grupo de pacientes descompensados el valor promedio de la edad es superior, no se encontró diferencia estadísticamente significativa, por lo que a pesar de la tendencia referida no se prueba en este estudio que exista asociación de la edad $(E T A=0,765)$ con la descompensación de la TA en el acto anestésico quirúrgico. Varios son los autores que han referido la edad avanzada como uno de los factores que deben tenerse en cuenta antes, durante y posterior al acto anestésico quirúrgico, resaltando que esto no constituye una contraindicación, pero sí una alerta para incrementar las precauciones $5,10,21$.

Como se ilustra en la tabla 3, de las 56 mujeres investigadas sólo se descompensaron 22, que representa el 39,3\% de las mismas, entretanto, de los 32 hombres considerados se descompensaron la misma cifra, pero esta constituye el $68,7 \%$ de la muestra analizada en este grupo, de modo que se demuestra la vinculación del sexo a la descompensación de la TA durante el TQ $(p=0,015)$, con mayor prevalencia el sexo masculino. 
Se ha señalado que la prevalencia es altamente variable y se ve incrementada en pacientes jóvenes, del sexo masculino que son fumadores, con excesivo consumo de alcohol y que presentan estrés laboral, entre otros factores ${ }^{13}$. Sin embargo en el año 2015 la tasa de prevalencia de HTA en Cuba fue mayor en el sexo femenino, así como el grupo etario de 60 a 64 años ${ }^{5}$.

Tanto el sexo como la raza han sido reportado en la literatura como factores de riesgo para la HTA, no modificables $5,7,13,14,22$, lo cual se hace más evidente, en este estudio, pues los recientes descubrimientos del mapa genético tienden a demostrar la participación de múltiples genes dentro del contexto de una predisposición genética multifactorial, entre los que destacan la expresión de los genes de la enzima convertidora de angiotensina (ECA), del gen del angiotensinógeno y la no expresión de la enzima sintetizadora de óxido nítrico endotelial 23,24 .

En otras investigaciones se ha encontrado también, una prevalencia mayor en el sexo masculino sobre el femenino, lo que se plantea es válido hasta los 50 años de edad. Después estas cifras tienden a investirse, al parecer por la privación de estrógeno que acompaña la menopausia ${ }^{5,22,25,26}$. Lo señalado, no constituye una regla que se cumpla bajo cualquier condición, ya que como se observa en los resultados de la presente investigación son múltiple los factores que influyen en la aparición de la HTA.

Al analizar la variable apariencia racial no se encontraron diferencias estadísticamente significativas. Se plantea que el color de la piel no es determinante en la aparición de la hipertensión arterial, aunque existen evidencias de que los individuos de color de piel negra sufren de complicaciones más graves, comienzan en edades tempranas de la vida y tienen mayor susceptibilidad a la lesión en órganos diana7,15.

Los resultados expuestos en la tabla 4, reflejan que los antecedentes patológicos personales juegan un papel importante en la descompensación de la TA durante el TQ (84,1\% vs. 15,9\%) destacándose particularmente las enfermedades renales, la epilepsia, las enfermedades cerebro vasculares (ECV), la diabetes mellitus y la cardiopatía isquémica, con 60\% o más; así como en los antecedentes personales familiares (APF) de hipertensión arterial.

Los resultados pueden deberse a que el individuo es portador del gen que determina la predisposición para una enfermedad determinada, según ha sido demostrado en distintos estudios de heredo patología, aunque se plantee una mayor probabilidad del padecimiento ${ }^{7,27,28,29}$ y además a que en el grupo sin antecedentes influyan otros factores de mayor importancia también aquí considerados.

En cuanto a la práctica de hábitos tóxicos (Tabla 5), las frecuencias relativas en los descompensados fueron superiores para todos los factores de riesgo, donde el tabaquismo ( $n=32$ ) y el cafeísmo ( $n=40)$ constituyeron el 72,7 y 90,9\%, respectivamente. Por esta razón, tanto el primer factor de riesgo

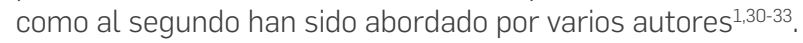

El consumo de bebidas alcohólicas se caracterizó en $79,5 \%(n=35)$ como bebedores sociales y la ingesta excesiva de sal en $36,4 \%$ (Tablas 5). Un mayor índice de masa corporal, una mayor circunferencia de la cintura, el tabaquismo y el alto consumo de alcohol se asocian de manera independiente con mayor PA sistólica, ${ }^{65-37}$. Por el contrario, el consumo leve a moderado de alcohol se ha asociado a un menor riesgo de muerte súbita cardíaca (MSC) en los varones ${ }^{34}$.

En la tabla 6, se ilustra que la hipercolesterolemia se presenta en 14 pacientes (31,8\%) descompensados, cifra mayor que en los compensados $(p<0,05)$. En la esfera emocional, se encontró ansiedad alta en 11 operados (25\%) que se descompensaron en el TQ; en tanto en el grupo de los compensados no hubo ninguno. Se identificó en 63,6\% vulnerabilidad severa al estrés ( $n=28)$ y un paciente con extrema vulnerabilidad en aquellos descompensados; mientras que en los no descompensados la frecuencia fue menor $(6,8 \%)$ y nula respectivamente.

Se destaca que factores de riesgo modificables como el tabaco, el consumo nocivo de alcohol, la dieta poco saludable, la insuficiente actividad física, la obesidad, así como la hipertensión, la hiperglucemia y el hipercolesterolemia, constituyen factores de riesgo principales que contribuyen a la patología subyacente de las enfermedades cardiovasculares ${ }^{1,32,38-44}$.

El término infarto agudo de miocardio, hace referencia a un riego sanguíneo insuficiente con lesión miocárdica, producido por una obstrucción en una o varias arterias coronarias. Acorde a los reportes de la Organización Mundial de la Salud (OMS), la exposición a estrés crónico hace más propensas a las personas a enfermar y morir de enfermedades cardiovasculares, además de doblar el riesgo de aparición de un infarto agudo miocárdico (IAM). La OMS pronostica que en el 2020 la enfermedad isquémica del corazón (EIC) será responsable de 11 millones de muertes, por lo que su prevención constituye un reto para las autoridades sanitarias de todos los países del mundo ${ }^{45-47}$. La evaluación preoperatoria y las alternativas durante el proceso de la anestesia son claves en la reducción de estos riesgos ${ }^{48-52}$.

Nutricionalmente en ambos grupos, los mayores porcentajes se ubicaron en la categoría normal (Tabla 7); sin embargo, con bajo peso, sobrepeso y obesos son más en el grupo de los descompensados con $n=8(18,2 \%)$ y $n=7$ (15,9\%), correspondientemente.

Algunos investigadores destacan haber encontrado dentro de las causales de la HTA el sedentarismo $0^{8,14,27,29}$, igualmente en este estudio, se encontró asociación entre este factor de riesgo y el mayor porcentaje de descompensación, 60,0 \%, en los sedentarios y $11,1 \%$ en los no sedentarios, durante el TQ. No se puede considerar que por sí solo, el sedentarismo constituya un elemento capaz de producir HTA. Se deben conjugar varios factores de riesgos como los antecedentes patológicos personales, la ansiedad, la vulnerabilidad al estrés y el tabaquismo que pueden influir en la descompensación hemodinámica de los pacientes. Según la literatura, existen muchos factores más que se conjugan para desencadenar la HTA, la que desencadena otros procesos que representan un gran peligro para la vida ${ }^{3,5,7,44,53}$.

Al analizar el riesgo, se obtuvo que influyen de forma determinante en el desequilibrio hemodinámico, el sexo masculino, los APP, el tabaquismo, cafeísmo y la hipercolesterolemia, dado valores de $\mathrm{OR}>1$ y al considerar el Intervalo de Confianza al $95 \%$ se tuvo que el 1 no cae en dichos intervalos; lo que representa que la probabilidad de descompensación de la TA durante el TQ aumenta (según el valor obtenido de OR) en presencia de los factores evaluados, por lo que constituyen riesgo.

Se ha demostrado otros efectos negativos sobre la morfología y hemodinámica cardiovascular inducidos por la nicotina y el humo de los cigarrillos, como la de inhibir la síntesis vascular y miocárdica de prostaciclina (PGI2) que unida al daño endotelial son factores de riesgo que predisponen a la hipertensión arterial, aterosclerosis, trombosis y alteraciones isquémicas $5,15,54,55$. 


\begin{tabular}{|c|c|c|c|}
\hline TENS & ARTERIAL & $\begin{array}{l}\text { Tensión arterial pr } \\
\text { pacientes que resul }\end{array}$ & tésica (mmHg) \\
\hline & & Descompensados & Compensados. \\
\hline Promedic & TA sistólica & 120 & 112 \\
\hline Desviació & stándar TA sistólica & 11 & 11 \\
\hline Promedic & TA diastólica & 77 & 70 \\
\hline Desviació & stándar TA diastólica & 8 & 9 \\
\hline ANOVA & TA SISTÓLICA & $\mathrm{p}=0,034$ & \\
\hline & TA DIASTÓLICA & $\mathrm{p}=0,001$ & \\
\hline
\end{tabular}

Fuente: Planilla Recopiladora de Datos

TA: Tensión Arterial TQ: Tiempo Quirúrgico

Tabla 1. Cifras de tensión arterial preanestésica de pacientes descompensados y compensados en el TQ.

\begin{tabular}{|l|l|c|c|}
\multicolumn{2}{c}{ EDAD } & \multicolumn{2}{c|}{ Elevación de la TA durante el TQ } \\
\cline { 3 - 4 } \multicolumn{2}{|c|}{} & SI $(\mathrm{n}=44)$ & NO $(\mathrm{n}=44)$ \\
\hline \multirow{2}{*}{ Años } & Media & 54 & 49 \\
\hline \multirow{3}{*}{ Años } & Desviación estándar & 12 & 16 \\
\cline { 2 - 4 } & Edad mínima & 28 & 19 \\
\cline { 2 - 4 } & Edad máxima & 78 & 81 \\
\hline \multirow{2}{*}{ Asociación } & Coeficiente ETA $=0,765$ & $\mathrm{p}>0.05$ \\
\hline
\end{tabular}

Fuente: Planilla Recopiladora de Datos

TA: Tensión Arterial TQ: Tiempo Quirúrgico

Tabla 2. Edad, su relación con las cifras de TA.

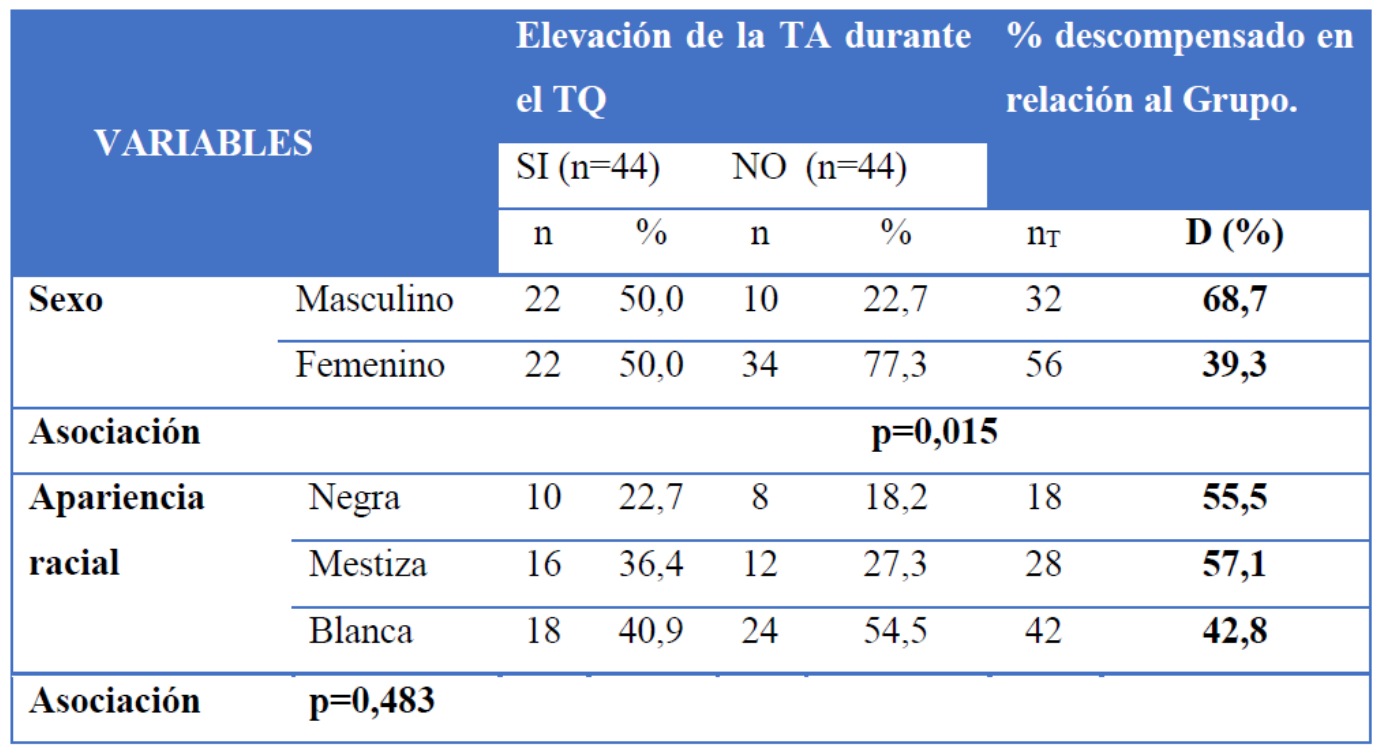

Fuente: Planilla Recopiladora de Datos

TA: Tensión Arterial TQ: Tiempo Quirúrgico

Tabla 3. Comportamiento de la TA según el sexo y la apariencia racial. 


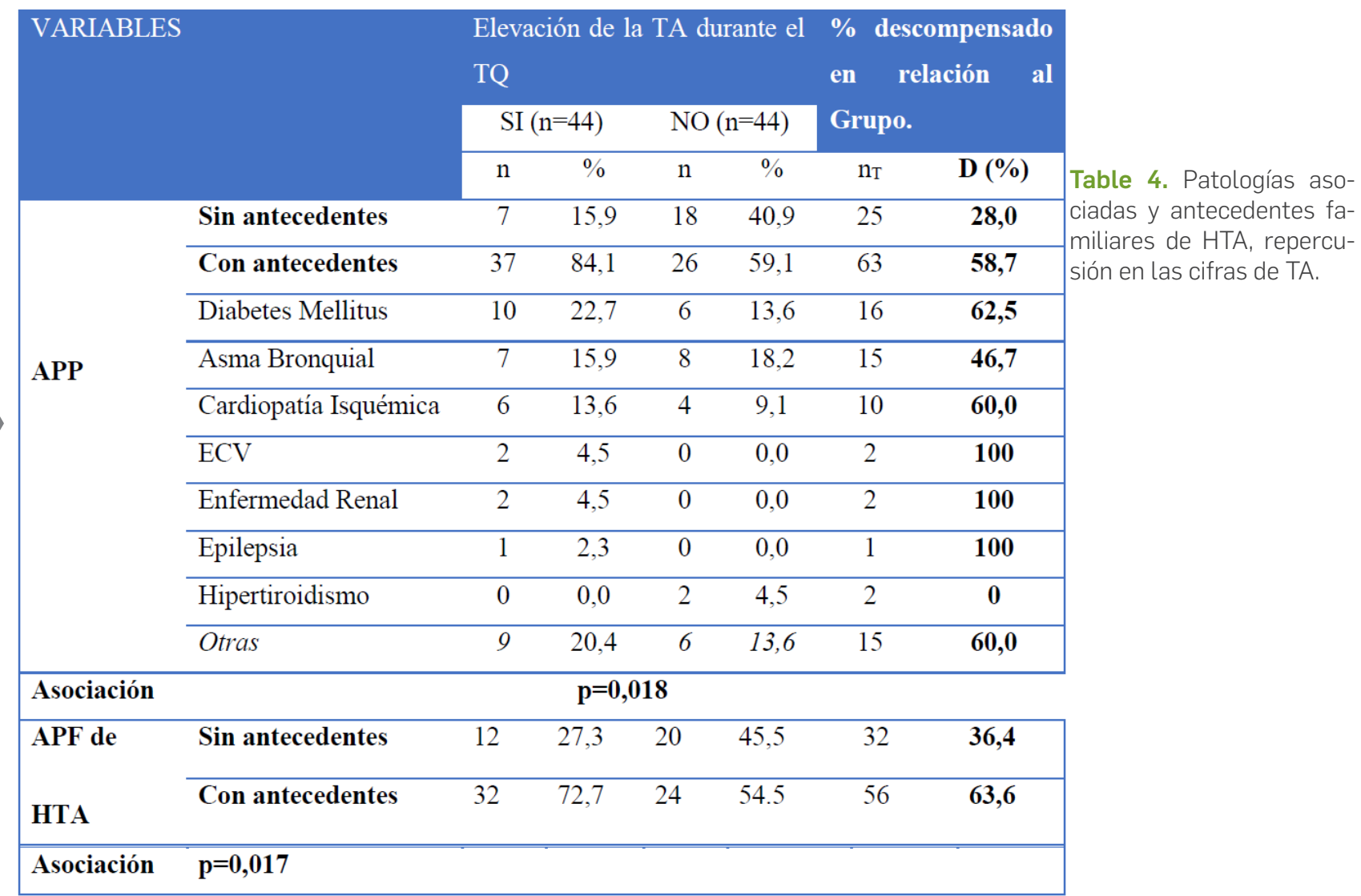

Fuente: Planilla Recopiladora de Datos

TA: Tensión Arterial, TQ: Tiempo Quirúrgico, APP: Antecedentes Patológicos Personales, ECV:

Enfermedad Cerebro Vascular, APF: Antecedentes Patológicos Familiares, HTA: Hipertensión Arterial

\begin{tabular}{|c|c|c|c|c|c|c|}
\hline \multirow{3}{*}{ VARIABLES } & & \multicolumn{3}{|c|}{ Elevación durante el TQ } & \multicolumn{2}{|c|}{$\%$ descompensado } \\
\hline & & $\mathrm{SI}(\mathrm{n}=44)$ & \multicolumn{2}{|c|}{$\mathrm{NO}(\mathrm{n}=44)$} & \multicolumn{2}{|c|}{$\begin{array}{l}\text { en relaciórn al } \\
\text { Grupo. }\end{array}$} \\
\hline & & n $\quad \%$ & $\mathrm{n}$ & $\%$ & $\mathrm{n}_{\mathrm{T}}$ & D (\%) \\
\hline \multirow[t]{2}{*}{ Tabaquismo } & $\mathrm{Si}$ & 3272,7 & 5 & 11,4 & 37 & 86,5 \\
\hline & No & $12 \quad 27,3$ & 39 & 88,6 & 51 & 23,5 \\
\hline Asociación & & $\mathrm{p}=\mathbf{0 , 0 0 0}$ & & & & \\
\hline \multirow[t]{2}{*}{ Cafeísmo } & $\mathrm{Si}$ & $40 \quad 90,9$ & 22 & 50,0 & 62 & 64,5 \\
\hline & No & $4 \quad 9,1$ & 22 & 50,0 & 26 & 15,4 \\
\hline Asociación & $\mathrm{p}=\mathbf{0 , 0 0 0}$ & & & & & \\
\hline \multirow{3}{*}{$\begin{array}{l}\text { Consumo de } \\
\text { bebidas } \\
\text { alcohólicas }\end{array}$} & Bebedor social & 3579,5 & 43 & 97,7 & 78 & 44,9 \\
\hline & $\begin{array}{l}\text { Consumo de } \\
\text { riesgo }\end{array}$ & $6 \quad 13,6$ & 1 & 2,3 & 7 & 85,7 \\
\hline & $\begin{array}{l}\text { Consumo } \\
\text { perjudicial }\end{array}$ & 36,8 & 0 & 0,0 & 3 & 100 \\
\hline Asociación & $\mathrm{p}=\mathbf{0 , 0 0 0}$ & & & & & \\
\hline \multirow[t]{2}{*}{$\begin{array}{l}\text { Consumo de } \\
\text { sal }\end{array}$} & Normal & $\begin{array}{ll}2 & 63,6 \\
8 & \end{array}$ & 42 & 95,5 & 70 & 40,0 \\
\hline & Exceso & $\begin{array}{ll}1 & 36,4 \\
6 & \end{array}$ & 2 & 4,5 & 18 & 88,9 \\
\hline Asociación & $\mathrm{p}=\mathbf{0 , 0 0 0}$ & & & & & \\
\hline
\end{tabular}

Tabla 5. Hábitos tóxicos y su relación con el comportamiento de la TA de los pacientes en estudio.

Fuente: Planilla Recopiladora de Datos

TA: Tensión Arterial TQ: Tiempo Quirúrgico 


\begin{tabular}{|c|c|c|c|c|c|c|c|}
\hline \multirow{3}{*}{\multicolumn{2}{|c|}{ VARIABLES }} & \multicolumn{4}{|c|}{$\begin{array}{l}\text { Elevación de la TA durante } \\
\text { el TQ }\end{array}$} & \multirow{2}{*}{\multicolumn{2}{|c|}{$\begin{array}{c}\% \\
\text { descompensado } \\
\text { en relación al Grupo. }\end{array}$}} \\
\hline & & \multicolumn{2}{|c|}{$\mathrm{SI}(\mathrm{n}=44)$} & \multicolumn{2}{|c|}{ NO $(n=44)$} & & \\
\hline & & & $\%$ & $\mathrm{n}$ & $\%$ & $\mathrm{n}_{\mathrm{T}}$ & $\mathrm{D}(\%)$ \\
\hline \multirow{2}{*}{\multicolumn{2}{|c|}{ Hipercolesterolemia }} & 14 & 31,8 & 4 & 9,1 & 18 & 77,8 \\
\hline & & 30 & 68,2 & 40 & 90,9 & 70 & 42,9 \\
\hline \multicolumn{2}{|l|}{ Asociación } & \multicolumn{4}{|c|}{$\mathrm{p}=0,017$} & & \\
\hline \multirow{4}{*}{ Ansiedad } & Muy baja & 0 & 0,0 & 8 & 18,2 & 8 & 0 \\
\hline & Baja & 17 & 38,6 & 30 & 68,2 & 47 & 36,2 \\
\hline & Normal & 16 & 36,4 & 6 & 13,6 & 22 & 72,7 \\
\hline & Alta & 11 & 25,0 & 0 & 0,0 & 11 & 100 \\
\hline \multicolumn{2}{|l|}{ Asociación } & \multicolumn{4}{|c|}{$\mathrm{p}=0,000$} & & \\
\hline \multirow[t]{4}{*}{ Estrés } & No vulnerable & 2 & 4,5 & 21 & 47,7 & 23 & 8,7 \\
\hline & Vulnerable & 13 & 29,5 & 20 & 45,5 & 33 & 39,4 \\
\hline & $\begin{array}{l}\text { Severamente } \\
\text { vulnerable }\end{array}$ & 28 & 63,6 & 3 & 6,8 & 31 & 90,3 \\
\hline & $\begin{array}{l}\text { Extremadamente } \\
\text { vulnerable }\end{array}$ & 1 & 2,3 & 0 & 0 & 1 & 100 \\
\hline \multicolumn{2}{|l|}{ Asociación } & \multicolumn{4}{|c|}{$\mathrm{p}=0,000$} & & \\
\hline
\end{tabular}

Fuente: Planilla Recopiladora de Datos

TA: Tensión Arterial TQ: Tiempo Quirúrgico

Tabla 6. Influencia del hipercolesterolemia, la ansiedad y el estrés en el estado hemodinámico del paciente quirúrgico.

\begin{tabular}{|c|c|c|c|c|c|c|c|}
\hline \multirow{3}{*}{\multicolumn{2}{|c|}{ VARIABLES }} & \multicolumn{4}{|c|}{ Elevación de la TA } & \multirow{2}{*}{\multicolumn{2}{|c|}{$\begin{array}{l}\% \\
\text { descompensado } \\
\text { en relación al } \\
\text { Grupo. }\end{array}$}} \\
\hline & & \multicolumn{2}{|c|}{$\mathrm{SI}(\mathrm{n}=44)$} & \multicolumn{2}{|c|}{$\mathrm{NO}(\mathrm{n}=44)$} & & \\
\hline & & $\mathrm{n}$ & $\%$ & $\mathrm{n}$ & $\%$ & $\mathrm{n}_{\mathrm{T}}$ & D (\%) \\
\hline \multirow{3}{*}{$\begin{array}{l}\text { Valoración } \\
\text { nutricional }\end{array}$} & Tormal & 29 & 65,9 & 38 & 86,4 & 67 & 43,3 \\
\hline & 3ajo peso & 8 & 18,2 & 4 & 9,1 & 12 & 66,7 \\
\hline & $\begin{array}{l}\text { obrepeso y } \\
\text { besos }\end{array}$ & 7 & 15,9 & 2 & 4,5 & 9 & 77,8 \\
\hline \multicolumn{2}{|l|}{ Asociación } & \multicolumn{6}{|c|}{$\mathrm{p}=\mathbf{0 , 0 1 7}$} \\
\hline \multirow[t]{2}{*}{ Sedentarismo } & $\mathrm{Si}$ & 42 & 95.5 & 28 & 63,6 & 70 & 60,0 \\
\hline & No & 2 & 4,5 & 16 & 36,4 & 18 & 11,1 \\
\hline
\end{tabular}

Fuente: Planilla Recopiladora de Datos

TA: Tensión Arterial TQ: Tiempo Quirúrgico

Tabla 7. Cifras de TA durante el TQ en relación a la valoración nutricional y sedentarismo. 


\section{Conclusiones}

Se pudo comprobar que los antecedentes patológicos personales y familiares, el tabaquismo, cafeísmo, sedentarismo y la hipercolesterolemia constituyen factores de riesgo de hipertensión arterial, que en la medida que se conjugan se produce una potenciación de sus efectos y se elevan considerablemente los riesgos de los pacientes operados en cirugía electiva, aun cuando estos sean considerados como normotensos en la evaluación preoperatoria, pues los que resultaron descompensados fueron los que entraron al salón con las cifras de tensión arterial normales, pero superiores.

\section{0}

\section{Referencias bibliográficas}

1. Williams B, Mancia G, Spiering W, Rosei EA, Azizi M, Clement DL. Guía ESC/ESH 2018 sobre el diagnóstico y tratamiento de la hipertensión arterial. Rev Esp Cardiol. 2019;72(2):160.e1-e78.

2. Fernández GL, Seuc JAH, Rodríguez GCA. Método de mortalidad ponderado según múltiples causas de muerte. Revista Finlay. 2019;9(3):197-209.

3. Ortiz R, Bermúdez V, Guzmán LJA, Silva PJS, Torres OMG, Carvajal GJS, Tirado REL, Cruz CTM, Espinoza DCl, Morocho AlA, Gratzia OM. Hipertensión arterial y su comportamiento epidemiológico en la población rural de Cumbe, Ecuador. Revista Latinoamericana de Hipertensión. 2019; 12(5):09-118.

4. Patel P, Ordunez P, DiPette D, Escobar MC, Trevor H, Wyss F, Hennis A, Asma S, Angell S. Mejor control de la presión arterial para reducir la morbilidad y mortalidad por enfermedades cardiovasculares: Proyecto de Prevención y Tratamiento Estandarizado de la Hipertensión Arterial. Rev Panam Salud Publica. 2017; 41:1-12.

5. González RR, Martínez CM, Castillo SD, Rodríguez MOL, Hernández VJ. Caracterización clínico-epidemiológica de la hipertensión arterial en adultos mayores. Revista Finlay. 2019; 7(2):74-80.

6. Sandoya E, Torres F, Rivedieu F, Machado F, Núñez E. Situación de la hipertensión arterial a nivel público y privado. Rev Urug Cardiol 2016; 31:219-227.

7. Rodríguez SA, Piña AAC, Díaz PA, García RR, Balcindes AS. Brechas en el manejo del paciente hipertenso en un área metropolitana de La Habana. Revista Finlay. 2019;9(4): 257-272.

8. Zuni ChKX, More SBE, Fernández VCD, García FBB, Ruiz OJM, Pérez RVK. Prevalencia de factores de riesgo cardiovascular en pacientes hospitalizados en un hospital de Lima. Rev. Fac. Med. Hum. 2019;19(4):68-73.

9. Soto EG, Moreno AL, Pahua DD. Panorama epidemiológico de México, principales causas de morbilidad y mortalidad. Revista de la Facultad de Medicina de la UNAM. 2016;59(6):8-22

10. Cuellar RS. Hipertensión Arterial. Ien líneal junio 2016. Disponible en: https://www.google.com/search? Y botplusweb.portalfarma. com. [Consultada 12 de febrero de 2020].

11. Rosas PM, Palomo PS, Borrayo SG, Madrid MA, Almeida GE, Galván OH, Magaña SJA, Saturno ChG. Consenso de Hipertensión Arterial Sistémica en México. Rev Med Inst Mex Seguro Soc. 2016;54 Supl 1:6-51.

12. León RC, Arroniz SR, Rodríguez-Cabello RS. Crisis hipertensivas. Atención primaria en la red. Guías clínicas; 2005;5(18).

13. López HMG, Meléndez FHJ, Álvarez RS, Alvarado AJL. Factores de riesgo para hipotensión en anestesia regional subaracnoidea para cesárea. Papel del Índice Cintura Cadera e Índice de Masa Corporal. Rev Colomb Anestesiol. 2018;46(1):45-51.

14. Sabio R, Valdez P. Recomendaciones latinoamericanas para el manejo de la hipertensión arterial en adultos (RELAHTA 2). Rev. virtual Soc. Parag. Med. Int. 2019; 6 (1):86-123.

15. Esteban FA, Fernández CA, Gómez de Diego JJ, Vicent AML, Vidal PR. Cardiología hoy 2018. Resumen anual de los avances en investigación y cambios en la práctica clínica. (C) 2018 Sociedad Española de Cardiología. Publicación oficial. Pág. 1105.
16. Bustamante R ¿ Qué Hacemos los Anestesiólogos? Desde la vigilancia anestésica monitorizada hasta la anestesia general. REV. MED. CLIN. CONDES - 2017; 28(5) 671-681.

17. Puerto LJA, Torres AFL, Cabanes RE. Herida por cuerpo extraño en el tórax. A propósito de un caso. Universidad y Sociedad. 2019;12(1):381-385.

18. Ronquillo GK, Rivera OA, Marín GAL. Análisis y aplicación de una lista de verificación en anestesia en trauma y cirugía de urgencia. Revista Mexicana de Anestesiología. 2019;42(1):56-61.

19. Del Pozo JH, Leiva SL. Hipertensión arterial y riesgo preoperatorio en el paciente anciano. Acta Médica; 2002 :(10):1-2.

20. Del Pozo JH, Leiva SL. Atención prequirúrgica del hipertenso eventual. Rev Cubana Med. 1998; 37(3):141-6.

21. Mille LJE, Ortiz MJJ, Rocha MJF. Manejo expectante de la hipertensión arterial transoperatoria: ¿es la anestesia la solución? Revista Mexicana de Anestesiología. 2017;40(Supl. 1):S90-S93.

22. Pomares AAJ, Vázquez NMA, Ruíz DES. Adherencia terapéutica en pacientes con hipertensión arterial. Revista Finlay. 2017;7(2):8-88.

23. Juárez IM, de la Fuente LMA, Fontecha AJ. Los nutrientes de la leche en la salud cardiovascular. Nutr Hosp. 2015;31(Supl. 2):26-32.

24. García RP, Gómez CR, Sánchez VN. Prevalencia de la Hipertensión Arterial y factores de riesgo presentes en la población de un área de Salud. Rev Méd Electrón; 2006:28(1).

25. Thierer $J$ ¿Otro escenario más para el canto del cisne de la aspirina? Estudio TWILIGHT. Rev Argent Cardiol 2019;87:517-520.

26. Rodríguez SPL, Alfonso HC, Valladares GA, Pomares AJ, López AL. Estrés y emociones negativas en adultos medios con hipertensión arterial esencial. Revista Finlay. 2018;8(1):36-45.

27. González RR. Factores de riesgo de hipertensión arterial en adolescentes. Revista Finlay. 2016; 6(4):262-264.

28. Costabel JP, Mandó F, Avegliano G. Miocardiopatía dilatada: ¿cuándo y cómo proceder a la investigación etiológica? Rev Urug Cardiol 2018:33:343-349.

29. Vázquez ME, Calderón RZG, Arias RJ, Ruvalcaba LJC, Rivera RLA, Ramírez ME. Sedentarismo, alimentación, obesidad, consumo de alcohol y tabaco como factores de riesgo para el desarrollo de diabetes tipo 2. Journal of negative and positive results. 2019;4(10):10111021.

30. Duin BA, Sosa CB, Hernández HR, Camacho C, Camacho JC. Factores de riesgo cardiovascular en adolescentes. Revista Venezolana de Salud Pública. 2018; 6(2):17-25.

31. Cabrera GL, Herrera CG, Díaz TO. Actividades físico-recreativas y su influencia en la disminución de los niveles de tensión arterial en mujeres hipertensas de 60 a 70 años. Revista Conrado. 2017;14(62):124-133.

32. Sánchez AAG, Bobadilla SME, Dimas AB, Gómez OM, González GG. Enfermedad cardiovascular primera causa de morbilidad en un hospital de tercer nivel. Revista Mexicana de Cardiología. Rev Mex Cardiol 2016;27(s3):s98-s102.

33. Borges ML, Álvarez IM, Barroso BR, Turro PA, de la Torre NE. Algunos de los nuevos factores predictores de riesgo cardiovascular Panorama Cuba y Salud. 2016;11(3):51-55.

34. Martínez PRM, Carrero VAM, Cisneros SLG, Chipi RY, Olivera MA, Izquierdo RE. Estudio retrospectivo de muerte súbita cardiovascular intrahospitalaria en el Hospital Enrique Cabrera Cossío. CorSalud. 2018;10(1):32-39.

35. Diéguez MM, Miguel SPE, Rodríguez HR, López BJ, Ponce de León D, Reyna CJL. Prevalencia de hipertrigliceridemia y factores de riesgo cardiovascular en estudiantes de la Universidad de Ciencias Médicas. Holguín, 2014-2015. Medisur. 2018;16(1):35-45.

36. Guamialamá MJ, Salazar DD. Evaluación antropométrica según el Índice de masa corporal en universitarios de Quito. Rev. Salud Pública. 2018;20(3):314-318.

37. Fernández PV. Respuesta presora al estrés como marcador pronóstico de hipertensión futura. Facultad de Medicina. Departamento de Medicina. Universidad Autónoma de Madrid, 2013.

38. Chaves G, Brítez N, Maciel V, Klinkhof A, Mereles D. Prevalencia de factores de riesgo cardiovascular en una población adulta ambulatoria urbana: estudio AsuRiesgo, Paraguay. Rev Panam Salud Publica. 2015;38(2):136-43. 
39. León RML, García ÁY, Álvarez HR, Morales PC, Regal CVM, González LHD. Influencia del estrés psicológico y la actividad física moderada en la reactividad cardiovascular. Revista Finlay. 2018;8(3): 224-232.

40.Román VJA, Vázquez MVH, Loera MJ, Cantú SON, Cervantes VDA, Bernabé ACC. Panorama epidemiológico del paciente con hipertensión arterial sistémica no controlada en una unidad de medicina familiar de Reynosa, Tamaulipas. Aten Fam. 2016;23(1):14-18.

41. Espinosa BAD. Hipertensión arterial: cifras para definirla al comenzar 2018. Revista Finlay. 2018;8(1): 66-74.

42. Vélez AC, Vidarte CJA. Efecto de un programa de entrenamiento físico sobre condición física saludable en hipertensos. Rev. Bras. Geriatr. Gerontol. 2016;19(2):277-288.

43. Herrera AP, Pacheco MJ, Valenzuela RG, Málaga G. Autoconocimiento, adherencia al tratamiento y control de la hipertensión arterial en el Perú: Una revisión narrativa. Rev Peru Med Exp Salud Pública. 2017;34(3):497-504

44. Espinoza AM, Bollini C. Cirugía de hombro en posición silla de playa: estrategias y recomendaciones para la prevención de complicaciones isquémicas. Rev Chil Anest 2020; 49: 114-124.

45. Moreno MG, Mejía MCS, Fernández NMI, Sánchez NR. Incidencia y manejo del infarto agudo miocárdico con elevación del segmento ST, Hospital José María Velasco Ibarra, 2014. Enfermería Investiga, Investigación, Vinculación, Docencia y Gestión- 2016;1(4):151-157.

46. Short $\mathrm{H}$. Isquemia miocárdica perioperatoria en cirugía no cardíaca. Anaesthesia Tutorial of the Week. 2018; www.wfsahq.org/resources/anaesthesia-tutorial-of-the-week.pp 1-8.

47. Kunze S. Evaluación preoperatoria en el siglo XXI. REV. MED. CLIN. CONDES. 2017;28(5):661-670.

48. Ronquillo GDK, Quezada MEL, Saltos MSE, González MSM. Alternativas a la intubación orotraqueal ante una vía aérea difícil. RECIMUNDO. 2020;4(1):26-38
49.Salazar DLC, Neira EFE. Terapia endovascular múltiple para el manejo del vasoespasmo cerebral refractario. Rev CES Med 2020;34(1):74-82.

50. Domínguez LB, Ortega CG, Díaz PA, Broullón DA. Incidencias y causas de la cancelación de cirugía en un hospital universitario, Barranquilla, Colombia. Revista Electrónica Trimestral de Enfermería. Enfermería Global. 2020(57):507-515.

51. Recart A. cirugía mayor ambulatoria. una nueva forma de entender la medicina quirúrgica. REV. MED. CLIN. CONDES. 2017; 28(5) 682690.

52. Coloma R. Manejo de vía aérea no difícil. Desde la ventilación con bolsa hasta intubación oro traqueal. REV. MED. CLIN. CONDES. 2017; 28(5) 691-700.

53. Salas P, González C, Carrillo D, Bolte L, Aglony M, Peredo S, Ibarra X, Rojo A, Delucchi A, Pinto V, Saieh C, Ceballos ML. Hipertensión arterial en la infancia. Recomendaciones para su diagnóstico y tratamiento. Parte 2. Rama de Nefrología Infantil, Sociedad Chilena de Pediatría. Rev Chil Pediatr. 2019;90(3):336-342.

54. López Farre A, Casado S. Heart failure, redox alterations and endothelial dysfunction. Hypertension. 2001; 38(6):1400-5.

55. Cote MC. Vascular nurse as a smoking cessation specialist. J Vasc Nurs. 2000; 18(2):47-53

Received: 15 Enero 2020

Accepted: 26 Marzo 2020 